\title{
Apoptotic volume decrease as a geometric determinant for cell dismantling into apoptotic bodies
}

\author{
R Núñez ${ }^{1,2}$, SM Sancho-Martínez ${ }^{1,2}$, JML Novoa $^{1,2}$ and FJ López-Hernández ${ }^{\star, 1,2,3}$
}

Apoptosis is a mode of cell death through which cells are dismantled and cell remains are packed into small, membrane-bound, sealed vesicles called apoptotic bodies, which are easy to erase by phagocytosis by neighbouring and immune system cells. The end point of the process is to cleanly eliminate damaged or unnecessary cells without disrupting the surrounding tissue or eliciting an inflammatory response. The apoptotic process involves a series of specific events including deoxyribonucleic acid and nuclear fragmentation, protease-driven cleavage of specific substrates, which inhibits key survival functions and reorganizes the cell's structure, externalization of molecules involved in phagocytosis, membrane blebbing and cell shrinkage. Apoptotic volume decrease (AVD) leading to cell shrinkage is a core event in the course of apoptosis, the biological meaning of which has not been clearly ascertained. In this article we argue that volume loss is a geometrical requisite for cell dismantling into apoptotic bodies. This is derived from the cell's volume-to-surface ratio. Indeed, package of the original cell volume into smaller membrane-sealed vesicles requires that either cell membrane surface increase or cell volume decrease. In this sense, AVD provides a reservoir of membrane surface for apoptotic body formation. The strategic situation of AVD in the time course of apoptosis is also discussed in the context of apoptotic body formation.

Cell Death and Differentiation (2010) 17, 1665-1671; doi:10.1038/cdd.2010.96; published online 13 August 2010

\section{Apoptosis, Volume Decrease and Cell Shrinkage}

Apoptosis is a form of programmed cell suicide in which damaged or unnecessary cells implicated in biological processes ranging from embryogenesis to ageing, from normal tissue homoeostasis to many human diseases, are discarded cleanly without affecting the surrounding cells and without unleashing an inflammatory or immune response. ${ }^{1,2}$ Apoptosis is a regulated and controlled mode of cell death with defined phenotypic and biochemical characteristics resulting in cell dismantling into smaller, membrane-bound, spheroid corpuscles called apoptotic bodies. Apoptotic bodies contain cytosol, degraded proteins and deoxyribonucleic acid (DNA), organelles, intracellular membrane structures and fragments of the nucleus, and prevent cell content to be poured into the extracellular space. Apoptotic bodies externalize eat-me signals in the form of specific molecules such as phosphatidylserine or certain membrane carbohydrates, which are critical for their recognition and phagocytosis by neighbouring and immune system cells. ${ }^{1,2}$

In addition to cell dismantling into apoptotic bodies, other hallmarks of apoptosis include (i) the onset of core signalling cascades leading to the activation of determined proteases (most prominently caspases) that cleave and inactivate specific target molecules related to key cellular processes and activate others required for the execution of apoptosis; (ii) internucleosomal DNA fragmentation, chromatin condensation and nuclear rupture; (iii) disruption and reorganization of the cytoskeleton and contractile proteins; and (iv) cell shrinkage. ${ }^{1,2}$ Apoptotic volume decrease (AVD) leading to cell shrinkage is generally an early event in the course of apoptosis. It was noticed in the initial studies describing the process of apoptosis as a universal characteristic of apoptotic cells. $^{3,4}$ The precise mechanisms underlying AVD are not fully understood. It is believed that $\mathrm{K}^{+} \mathrm{Cl}^{-}$efflux and $\mathrm{Na}^{+}$ movements drive water extrusion off the cell. Two stages of AVD have been described, with distinct roles in the process of apoptosis. ${ }^{5-7}$ The first stage is characterized by a reversal of the normal $\mathrm{Na}^{+}$and $\mathrm{K}^{+}$gradients, leading to $\mathrm{Na}^{+}$accumulation and $\mathrm{K}^{+}$extrusion. It is estimated that these electrolytic movements are responsible for $20-40 \%$ of the cell volume decrease, which occurs before cytochrome $c$ release from the mitochondria. ${ }^{6} \mathrm{~A}$ second, independent stage of volume loss (to $80-85 \%$ ) depends on correct cytoskeleton organization and also relies on further $\mathrm{K}^{+}$

\footnotetext{
${ }^{1}$ Unidad de Fisiopatología Renal y Cardiovascular, Departamento de Fisiología y Farmacología, Universidad de Salamanca, Salamanca, Spain; ${ }^{2}$ Instituto Reina Sofía de Investigación Nefrológica, Fundación Renal Înigo Álvarez de Toledo, Madrid, Spain and ${ }^{3}$ Unidad de Investigación, Hospital Universitario de Salamanca, Salamanca, Spain

*Corresponding author: FJ López-Hernández, Unidad de Investigación, Hospital Universitario de Salamanca, Paseo de San Vicente, 58-182, Salamanca 37007, Spain. Tel: + 34923294 472; Fax: + 34923294 669; E-mail: flopezher@usal.es

Keywords: apoptotic volume decrease; volume-to-surface ratio; apoptotic bodies

Abbreviations: ATP, adenosine triphosphate; AVD, apoptotic volume decrease; Bcl-2, B-cell lymphoma 2; DNA, deoxyribonucleic acid; $\delta$, volume-to-surface area ratio; $r$, radius; ROCK-I, Rho-associated coiled-coil-forming kinase I; RT, room temperature; $S$, surface area; $V$, volume; Z-VAD-fmk, $N$-benzyloxycarbonyl-valinealanine-aspartic acid-fluoromethylketone

Received 26.2.10; revised 14.6.10; accepted 30.6.10; Edited by JA Cidlowski; published online 13.8 .10
} 
extrusion. Different cytoskeleton-disrupting agents, such as cytochalasins $B, D$ and $E$ and latrunculin $A$, prevent this second round of volume loss, along with DNA degradation and formation of apoptotic bodies. ${ }^{7,8}$ In this second stage, both cytochrome $c$ release and caspases seem to be involved and required for AVD (reviewed in Remillard and Yuan ${ }^{6}$ ). Yet, a channel-mediated $\mathrm{K}^{+}$outward movement is activated by cytochrome

$c$ independently of initiator caspase- 9 activation. ${ }^{9}$ However, the specific channels and transporters implicated in the electrolytic movements, their specific modulators during apoptosis and their effect on downstream apoptotic effectors have not yet been univocally unravelled, and they fall out of the scope of this article. Indeed, there are some indications suggesting that the channels behind AVD (i) might be different from cell type to cell type, and (ii) might be redundant to ensure AVD under a variety of external and internal scenarios. This reinforces the important role of AVD in apoptosis. ${ }^{10-12}$

Experimental prevention of AVD inhibits the execution of apoptosis and even cell death in most cell types. ${ }^{10-12}$ However, it is still unclear whether AVD is per se a primary activator of the apoptotic machinery, or is another consequence of the electrolytic imbalance occurring early in the apoptotic process. ${ }^{13-16}$ Independently of whether AVD is a cause or a consequence, this article intends to show that
AVD provides a solution for a geometrical requirement of cell dismantling into apoptotic bodies. In fact, inhibition of AVD with mechanistically different manoeuvres prevents apoptotic body formation (see below). Hitherto, research on AVD has been mainly addressed towards understanding the underlying mechanisms and towards establishing a cause-effect relationship with apoptosis and with other apoptotic events. However, speculations on potential teleological functions of AVD have not been put forward yet. In this article, we show that, for geometry's sake, decomposition of a sphere into smaller ones under constant surface area conditions requires that a part of the volume be lost during the process. This rule has a direct parallelism with round cell (e.g. lymphocyte) dismantling into apoptotic bodies. The same principle is also discussed and extended over non-spherical forms and cells.

\section{AVD is Required for Cell Dismantling into Apoptotic Bodies}

The mathematical relationship between the volume $(V)$ and surface area $(S)$ of a sphere $(\delta$; the number of $V$ units enclosed in each $S$ unit, or volume-to-surface ratio) equals the radius $(r)$ divided by 3 (Figure 1). It follows that the shorter the radius the lower the $\delta$. Consequently, the total volume contained in any number of spheres whose total surface area equals that of a

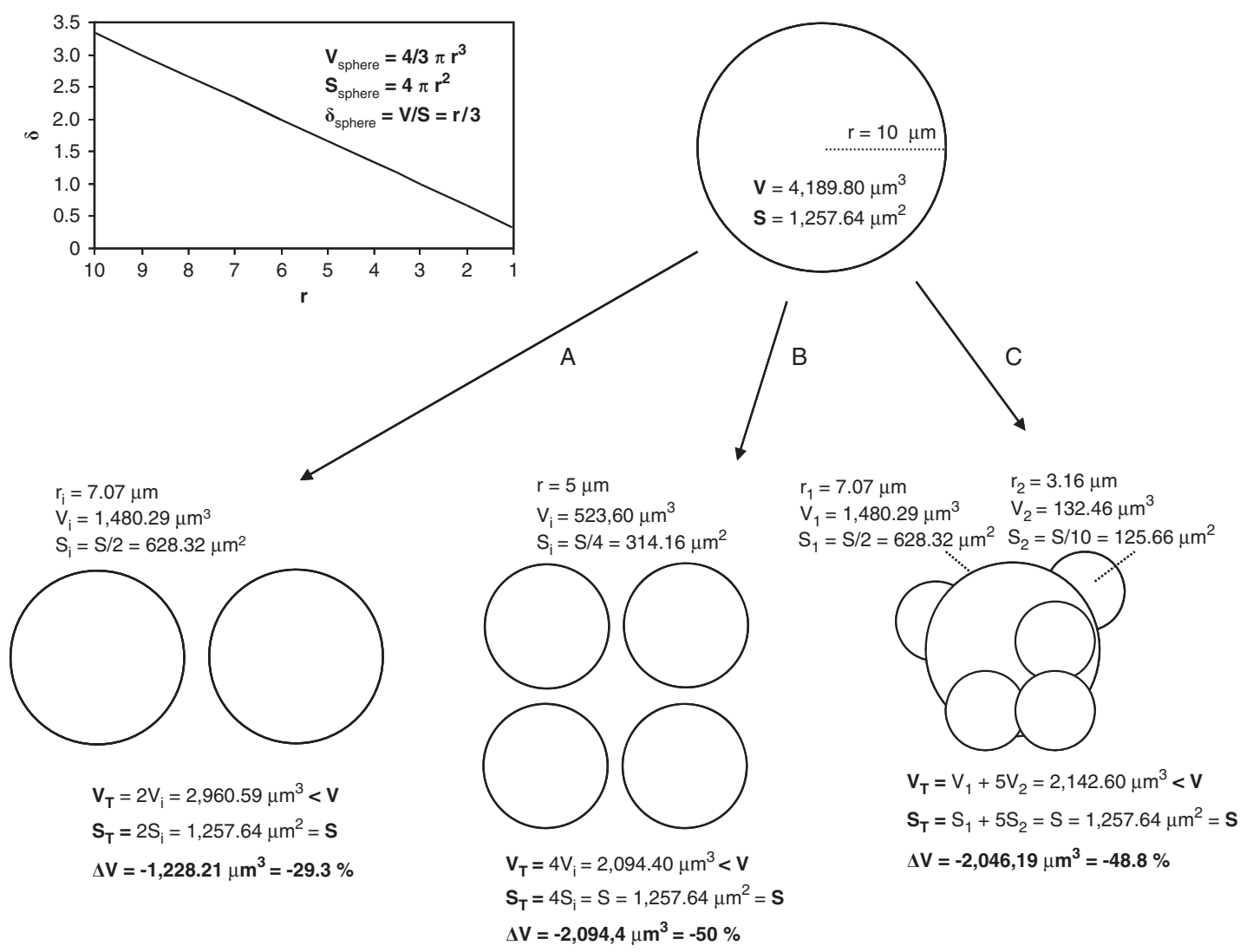

Figure 1 Graphical representation of the sphere's volume-to-surface ratio, and schematic depiction of the fragmentation of a sphere into smaller ones. Three possibilities are shown for illustrative purposes. In all cases, the total surface area of the smaller spheres equals the surface area of the initial one. A, two identical spheres; B, four identical spheres; and $\mathrm{C}$, one sphere as in A, plus 5 smaller ones. In all cases, the total volume enclosed by the fragmented spheres is smaller than that of the initial one. $\mathbf{r}=$ radius; $\mathbf{S}=$ surface area; $\mathbf{V}=$ volume; $\Delta \mathbf{V}=$ change in volume. Underscript $' i=$ of each individual sphere; Underscript ' $T$ ' $=$ total, of all spheres 


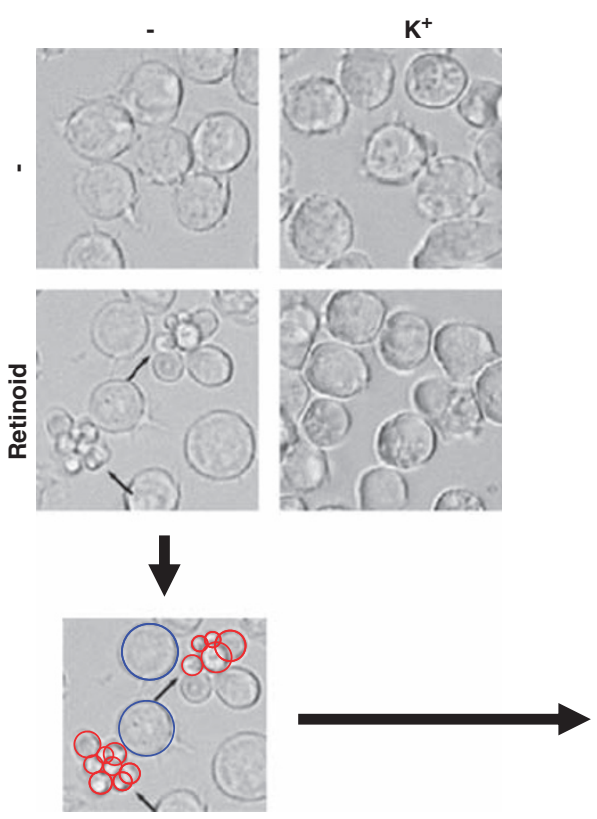

$$
\begin{array}{ll}
\mathrm{V}_{1}=4.18 \mathrm{au}^{3} & \mathrm{~S}_{1}=12.56 a u^{2} \\
\mathrm{~V}_{\mathrm{e}}=\Sigma_{2}^{6} \mathrm{~V}_{\mathrm{i}}=1.69 a u^{3} & \mathrm{~S}_{\mathrm{e}}=\Sigma_{2}^{6} \mathrm{~V}_{\mathrm{i}}=10.93 \mathrm{au}^{2} \\
\Delta \mathrm{V}=-59 \% & \Delta \mathrm{S}=-13.04 \%
\end{array}
$$
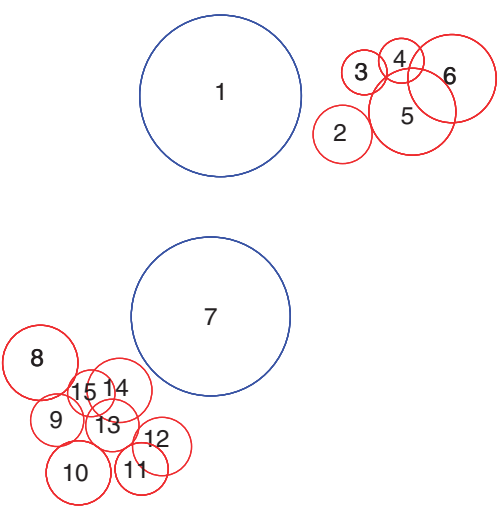

$$
\begin{aligned}
& \mathrm{V}_{7}=4.18 \mathrm{au}^{3} \\
& \mathrm{~V}_{\mathrm{e}}=\Sigma_{8}^{15} \mathrm{~V}_{\mathrm{i}}=1.78 \mathrm{au}^{3} \\
& \Delta \mathrm{V}=-57 \%
\end{aligned}
$$

$$
\begin{aligned}
& S_{7}=12.56 a^{2} \\
& S_{e}=\Sigma_{8}^{15} V_{i}=13.94 a u^{2} \\
& \Delta S=10.94 \%
\end{aligned}
$$

Figure 2 Representative images of Jurkat T cells treated with vehicle (-) or a pro-apoptotic retinoid, in the presence or absence of a normal (-) or high concentration $(+60 \mathrm{mM})$ of extracellular potassium $\left(\mathrm{K}^{+}\right)$in the culture medium. Whereas fragmentation into apoptotic bodies is evident upon treatment with the retinoid, no signs of this process are evident when cells are treated with the retinoid in high $\mathrm{K}^{+}$-containing medium. As an approximation, the volume and surface area of apoptotic bodies appearing in the picture were quantified and compared to those of a normal cell. For this purpose, images were magnified and apoptotic bodies (red) or normal cell contours (blue) were represented by circles of most approximate size. The diameter of these circles was measured (in arbitrary units of length, au), and the volume (V) and surface area (S) of the corresponding spheres were calculated. It is evident that the approximate volume of the apoptotic bodies $\left(\mathbf{V}_{\mathrm{e}}\right)$ totalling a surface area $\left(\mathbf{S}_{\mathrm{e}}\right)$ similar to that of a normal cell $\left(\mathbf{S}_{1}\right.$ or $\left.\mathbf{S}_{7}\right)$ is significantly lower than the volume of a normal cell. $\Delta \mathbf{S}=$ percentage change in surface area $=\left(S_{e}-S_{1}\right.$ or 7$) \times 100 / S_{1}$ or $7 ; \Delta \mathbf{V}=$ percentage change in volume $=\left(\mathrm{V}_{\mathrm{e}}-\mathrm{V}_{1}\right.$ or 7$) \times 100 / \mathrm{N}_{1}$ or 7

larger one is lower than the volume of the large sphere (Figure 1). In other words, decomposition of a sphere into smaller ones requires either an increase in the total surface area or a decrease in the total volume contained. Lymphocytes and, in general, non-attached cells are spheroid cells whose dismantling into apoptotic bodies clearly resembles this situation. In order to comply with these geometrical determinants, either cell volume must grow lower or cell membrane surface area must grow larger. Among other potential functions, AVD provides a solution to spheroid cell dismantling. Figure 2 exemplifies the case of Jurkat cell dismantling into apoptotic bodies after treatment with a retinoid. Increasing extracellular potassium prevents AVD, membrane blebbing and cell fragmentation. For illustrative purposes, the total volume and surface area of apoptotic bodies formed in Jurkat cells are measured (as indicated in the figure legend) and compared with the volume and surface area of an average non-apoptotic cell. It can be seen that the volume wrapped in apoptotic bodies totalling the same surface area as that of a native cell is much smaller than the volume of the normal cell.

The case of non-spherical cells is not so straightforward, although the same principles can be applied with some clarification. For simplicity, the case of a parallelepiped undergoing decomposition into spheres is depicted in the poster (Figure 3). The sphere is the geometrical figure with the highest $\delta$; the sphere has the smallest surface area among all surfaces enclosing a given volume and it encloses the largest volume among all closed surfaces with a given surface area. Accordingly, a parallelepiped cannot be transformed into a sphere with the same volume and surface area (Figure 3). However, a parallelepiped can be mathematically decomposed in a determined number of spheres, the whole volume of the parallelepiped being exactly divided into the spheres, and the sum of the surface area of the spheres equalling the surface area of the parallelepiped, as long as each sphere has the same $\delta$ as the parallelepiped. Beyond this threshold, decomposition of the parallelepiped into a higher number of smaller spheres requires volume reduction or an increase in surface area. As all investigators in cell biology know, following detachment all cells become spheroids. This is an advantage for the package of fragments, because of the sphere having the lowest $\delta$ of all geometrical figures. Again, AVD provides a solution to polygonal cell dismantling into small, spheroid apoptotic bodies.

Interestingly, experimental data support a relation between AVD and apoptotic body formation. Indeed, suppression of apoptotic sodium accumulation fails to inhibit several downstream apoptotic events initiated by Fas stimulation, such as phosphatidylserine externalization, caspase activation, poly-(adenosine diphosphate)-ribose polymerase cleavage, chromatin condensation and internucleosomal DNA fragmentation; but completely abolishes cell shrinkage (cells even swell) and apoptotic body formation. ${ }^{16}$ This suggests that AVD and apoptotic body formation might be mechanistically related. Another link between AVD and apoptotic body 


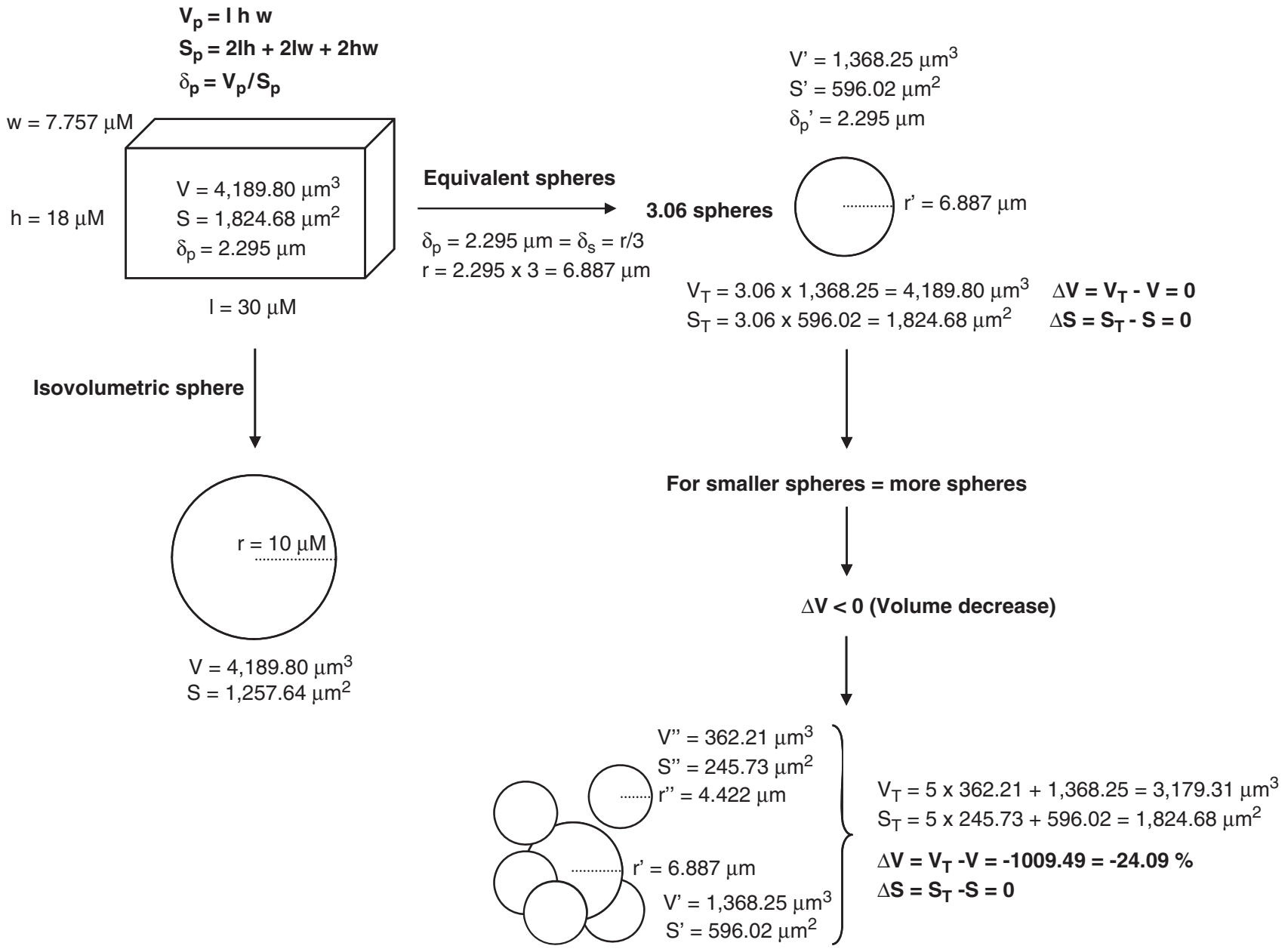

Figure 3 Graphical representation of the decomposition of a parallelepiped into spheres. Because the sphere is the geometrical figure with the highest volume-to-surface area ratio $(\delta)$, the sphere enclosing the same volume as a determined parallelepiped has a lower surface area. A parallelepiped can be mathematically decomposed in a number of spheres, each of which having the same $d$ as the parallelepiped, totalling the same volume and surface area as the said parallelepiped. This size of sphere settles the threshold for $\delta$. If the parallelepiped were divided into smaller spheres, their additive volume fitting in the total surface area would be lower than that of the initial parallelepiped. $\mathbf{h}=$ height; $\mathbf{I}=$ length; $\mathbf{r}=$ radius; $\mathbf{S}=$ surface area; $\mathbf{S}_{\mathbf{p}}=$ surface area of the parallelepiped; $\mathbf{S}_{\mathbf{T}}=$ total surface area of all spheres; $\mathbf{V}=$ volume; $\mathbf{V}_{\mathbf{p}}=$ volume of the parallelepiped; $\mathbf{V}_{\mathbf{T}}=$ total volume of all spheres; $\mathbf{W}=$ width; $\Delta \mathbf{V}=$ change in volume

formation is provided by the action of inhibitors of cytoskeleton organization. Cytoskeletal disruption inhibits the second stage of AVD and also apoptotic body formation. ${ }^{7}$ As far as we know, these experimental manoeuvres do not exist in nature, but serve to unravel and support a connection between AVD and apoptotic body formation.

\section{The Apoptotic Network as an Integral Mechanism of Control}

Apoptotic body formation is thought to be the intermediate goal of cells committed to dying, which is directed towards facilitating the end point of the process, namely the clean disappearance of the cell remains by phagocytosis. ${ }^{1,2}$ As such, the apoptotic machinery targets specifically, rather than indiscriminately, key cellular structures and functions so that cell viability is disrupted, and cell content is fragmented and packed in small, sealed vesicles readily marked for phagocytosis. $^{17}$ Apoptosis as a whole is an active, adenosine triphosphate (ATP)-consuming process ${ }^{18}$ during which certain cellular functions are preserved and reoriented towards the new fate. Among other substrates, caspases cleave proteins forming part of the cellular cytoskeleton and focal adhesion complexes, leading to (i) cytoskeleton rearrangement towards securing the apoptotic phenotype, and (ii) cell detachment from surrounding cells and basement membranes, as a landmark of the apoptotic course towards cell dismantling. ${ }^{1}$

Cytoskeleton rearrangement enables cell membrane blebbing, another hallmark of apoptosis. Blebbing is intrinsically related to the formation of apoptotic bodies. Indeed, membrane protrusions originated by blebbing are considered to be the progenitors of apoptotic bodies. ${ }^{1,19}$ Blebbing (also named zeiosis) occurs in two stages. Interestingly, the first one occurs only in attached cells. In these cells, $\delta$ is, in all cases, lower than the $\delta$ of the sphere (or round cell upon detachment) enclosing the same cell volume, under a cytosol volume conservation principle supported by 
experimental evidence, and mathematically modelled under an energy minimization assumption. ${ }^{20}$ As a consequence, upon rounding, cell surface is in excess for the volume. ${ }^{19}$ This type of blebbing is also seen in cells undergoing important structural rearrangements, such as division, migration, etc. ${ }^{19}$ However, the mechanisms of this blebbing may be different from those underlying the formation of apoptotic blebs. Different types of blebs have been recognized, involving distinct participation of the different elements of the cytoskeleton and their connection with the plasmalemma. ${ }^{21}$ Still, in all situations coursing with blebbing, the cell's shape changes, and thus blebbing-mediated crumpling of the cell membrane may serve to accommodate $\delta$. As such, ruffling of the membrane by blebbing provides a more than coincidental reconciliation for volume and surface area during the detachment phase of apoptosis until division into smaller fragments.

In apoptosis, membrane blebbing is originated by microtubule disassembly and actin polymerization in the cortical microfilament network. This actin polymerization forms restriction rings for bleb enucleation and formation, and enables actin-myosin-driven contraction. ${ }^{22,23}$ Contraction creates intracellular pressure and provides surface isometric tension for membrane herniation within the restriction rings, with the size of the bleb being proportional to tension. ${ }^{23}$ Actomyosin contraction is activated by phosphorylation $^{24}$ mediated by the Rho kinase isoform Rho-associated coiled-coil-forming kinase I (ROCK-I), which in turn is cleaved and activated by caspases during apoptosis. ${ }^{19}$ However, other activators of ROCK-I must exist during apoptosis, since some cell types may bleb for long periods of time in the presence of caspase inhibitors. ${ }^{24}$ ROCK activity is necessary and sufficient for the formation of membrane blebs, and participates in myosin ATPase activity and in the coupling of actin-myosin filaments to the plasma membrane. The second round of blebbing occurs in all types of cells. It is partly dependent on ROCK activity and intimately related to apoptotic body formation. In fact, incubation of apoptotic cells with ROCK-I inhibitors inhibits cell fragmentation, but not other apoptotic events such as DNA fragmentation. ${ }^{19}$

Membrane blebs pinching off the cell's main body by strong contraction give rise to independent apoptotic bodies. The release of bleb vesicles is a protease-dependent process, in which caspases seem to have a central role. ${ }^{24,25}$ It has been shown that caspase inhibition prevents the release of apoptotic bodies induced by extrinsic and intrinsic pathway activators, but not their formation by blebbing. ${ }^{25}$ Membrane blebbing induced by retinoids (activators of the intrinsic pathway) and Fas stimulation (extrinsic pathway) is completely abolished by stable overexpression of B-cell lymphoma 2 (Bcl-2). However, inhibition of caspase 9 by stably overexpressing a catalytically inactive mutant of this caspase prevents apoptotic body release but only partially inhibits membrane blebbing induced by all these stimuli. ${ }^{25}$ In addition, the pan caspase inhibitor $N$-benzyloxycarbonyl-valine-alanineaspartic acid-fluoromethylketone (Z-VAD-fmk) completely inhibits blebbing and apoptotic body release induced by Fas stimulation, but only the apoptotic body release induced by retinoids.
These results indicate that the apoptotic signal must reach the mitochondria for membrane blebbing to occur, whereas the release of apoptotic bodies (bleb individualization) is prevented by all manoeuvres inhibiting the activation of executioner caspases. In the case of Fas stimulation, both Z-VAD-fmk (by inhibiting the apoptotic signal very apically, at the level of caspase 8/10) and Bcl-2 overexpression (by inhibiting mitochondrial depolarization and hyper permeability) prevent not only apoptotic body release, but also membrane blebbing and cell death. However, inhibition of caspase 9 (and downstream executioner caspase 3) prevents Fas-induced apoptotic body release but not blebbing. ${ }^{25}$ However, in the case of retinoids, the apoptotic signal upstream of mitochondria is caspase-independent. ${ }^{26}$ That is why the pan caspase inhibitor Z-VAD-fmk cannot block retinoid-induced blebbing, but prevents the executioner caspase-dependent apoptotic body release. The whole process of apoptotic execution is active at room temperature (RT; 19-25 $\mathrm{C}$ ), including membrane blebbing, except apoptotic body release. ${ }^{27}$ Because caspases are normally active at $\mathrm{RT},{ }^{27}$ there is a caspase-independent, temperaturedependent step in the process of apoptotic body release, which remains to be determined.

Most importantly, coinciding with the decisive role of AVD in securing the apoptotic goal, it might be considered that AVD plays a sentry position in the regulation of downstream events. More accurately, the electrolytic movements occurring early in the apoptotic process control both AVD and other downstream events. ${ }^{6,7}$ Inhibition of $\mathrm{K}^{+}$extrusion by increasing $\mathrm{K}^{+}$extracellular concentration, or by means of $\mathrm{K}^{+}$channel blockers, prevents all or the most relevant downstream events from taking place, including AVD and cytochrome $c$ translocation. ${ }^{28,29}$ The latter is inhibited even in cells stimulated for apoptosis with insults, whose mechanisms of action do not require caspases for cytochrome $c$ release. This is not a trivial issue because mitochondrial injury has been proposed as the no-return point in the commitment of cells to apoptosis. ${ }^{30,31}$

Furthermore, both caspases and several nucleases responsible for apoptotic DNA fragmentation remain inhibited under the normal intracellular concentration of $\mathrm{K}^{+}\left(\mathrm{K}_{\mathrm{i}}^{+}\right)$, whereas they become active when $\mathrm{K}_{\mathrm{i}}^{+}$lowers. ${ }^{10,32}$ This is a biochemical property, as it has been corroborated in vitro with purified enzymes subject to different $\mathrm{K}^{+}$concentrations. ${ }^{32}$ Apoptosis protease-activating factor-1-cytochrome $c$-procaspase 9 apoptosome formation is also inhibited at physiological $\mathrm{K}_{\mathrm{i}}^{+}{ }^{33}$ In addition, there seems to be a direct link between AVD and apoptotic body formation, independently from the control of other apoptotic events, and also independently from $\mathrm{K}^{+}$extrusion. As explained above, inhibition of apoptotic $\mathrm{Na}^{+}$entry also prevents AVD and apoptotic body formation, without affecting the development of the other typical characteristics of apoptosis, or the activity of caspases and nucleases. This indicates that apoptotic body formation is controlled from the first stage of AVD.

\section{Final Remarks}

At the organism level, the most important aspect of apoptosis is that cell death occurs without the release of molecules with the potential to unleash inflammation or cause injury to 
neighbouring cells. The end point of the apoptotic execution phase is to insure that the dying cell is appropriately packaged to avoid homeostatic havoc. In the model we propose, AVD is a predetermined and key event that, among other functions, allows the apoptotic cell to be fragmented into small apoptotic bodies by reducing the volume that has to be packed in vesicles, whose $\delta$ decreases with size. As commented, for spheroid (nonattached) cells this is a geometric requirement in all cases, whereas for attached cells it becomes necessary for division beyond a threshold of size. In all cases, cell volume shrinkage endows the cell membrane surface with the capacity to form an a priori undetermined number of small, sealed vesicles in which to pack intracellular debris for a clean disposal, in the case that they need to be formed in the process of cell dismantling. In a view, AVD provides a reservoir of $\delta$ to be used or not by the apoptotic cell, and the potential to decide on an individual basis the size and number of apoptotic bodies, depending on the course of other cell death-related events. For instance, the apoptotic cell often culminates into one single, shrunken corpse, or is completely split into multiple small apoptotic bodies, whereas also frequently it is fragmented into a large- or mediumsized body containing (or not) the nucleus and a variety of smaller ones. ${ }^{1}$

We also speculate that AVD is an early process during apoptosis ${ }^{10}$ partly because extrusion of volume out of the cell is an energy-consuming process. ${ }^{34}$ In fact, energy-depleted cells swell rather than shrink and dye through necrosis rather than apoptosis. ${ }^{35,36}$ Necrotic volume increase is caused by dysfunction of regulatory volume response due to impairment of volume-sensitive $\mathrm{Cl}^{-}$channels under conditions of ATP deficiency. ${ }^{34}$ In the course of apoptosis, the cell's energetic status must be preserved initially to some extent in order for the apoptotic machinery to work properly. ${ }^{18,37,38} \mathrm{~A}$ rapid energetic crisis aborts apoptosis and turns cell death into a necrotic-like phenotype. If AVD were left to later stages of the apoptotic course, at some point and under determined circumstances it might be compromised along with the possibility of fragmenting into small apoptotic bodies, or fragmenting at all in the case of non-attached cells.

Given the important role of apoptosis not only in development and homeostasis but also in human disease, the so-called cytoplasmic events of the apoptotic course gain special importance as determinants of the final fate of the cell and of the disposal of cell remains. ${ }^{1}$ Thus, further knowledge on these processes may also be of clinical interest. Precisely, aborted apoptosis or failed phagocytosis of the apoptotic cell ultimately leads to membrane disruption, release of content and activation of an inflammatory response. ${ }^{39,40}$ Inappropriate or defective cytosolic events leading to failure of cell dismantling into apoptotic bodies may lead to a less effective phagocytosis and inflammatory damage potentially contributing to the appearance or magnification of pathological processes, including autoimmune diseases. ${ }^{17}$ An in-depth knowledge of the precise mechanisms underlying these processes, and those failing in disease or being altered by pathological circumstances, may help to design new therapeutic strategies. ${ }^{6}$

\section{Conflict of interest}

The authors declare no conflict of interest.
1. Mills JC, Stone NL, Pittman RN. Extranuclear apoptosis. The role of the cytoplasm in the execution phase. J Cell Biol 1999; 146: 703-708.

2. Doonan F, Cotter TG. Morphological assessment of apoptosis. Methods 2008; 44 200-204.

3. Kerr JF, Wyllie AH, Currie AR. Apoptosis: a basic biological phenomenon with wide-ranging implications in tissue kinetics. Br J Cancer 1972; 26: 239-257.

4. Hengartner MO. The biochemistry of apoptosis. Nature 2000; 407: 770-776.

5. Klassen NV, Walker PR, Ross CK, Cygler J, Lach B. Two-stage cell shrinkage and the OER for radiation-induced apoptosis of rat thymocytes. Int J Radiat Biol 1993; 64: $571-581$.

6. Remillard CV, Yuan JX. Activation of K+ channels: an essential pathway in programmed cell death. Am J Physiol Lung Cell Mol Physiol 2004; 286: L49-L67.

7. Bortner CD, Sifre MI, Cidlowski JA. Cationic gradient reversal and cytoskeletonindependent volume regulatory pathways define an early stage of apoptosis. J Biol Chem 2008; 283: 7219-7229.

8. Levee MG, Dabrowska MI, Lelli Jr JL, Hinshaw DB. Actin polymerization and depolymerization during apoptosis in HL-60 cells. Am J Physiol 1996; 271: C1981-C1992.

9. Platoshyn O, Zhang S, McDaniel SS, Yuan JX. Cytochrome $c$ activates $K+$ channels before inducing apoptosis. Am J Physiol Cell Physiol 2002; 283: C1298-C1305.

10. Yu SP, Choi DW. Ions, cell volume, and apoptosis. Proc Natl Acad Sci USA 2000; 97 9360-9362.

11. Bortner CD, Cidlowski JA. Apoptotic volume decrease and the incredible shrinking cell. Cell Death Differ 2002; 9: 1307-1310.

12. Bortner CD, Cidlowski JA. The role of apoptotic volume decrease and ionic homeostasis in the activation and repression of apoptosis. Pflugers Arch - Eur J Physiol 2004; 448: 313-318.

13. Friis MB, Friborg CR, Schneider L, Nielsen MB, Lambert IH, Christensen ST et al. Cell shrinkage as a signal to apoptosis in NIH 3T3 fibroblasts. J Physiol 2005; 567: 427-443.

14. Orlov SN, Pchejetski D, Taurin S, Thorin-Trescases N, Maximov GV, Pshezhetsky AV et al. Apoptosis in serum-deprived vascular smooth muscle cells: evidence for cell volumeindependent mechanism. Apoptosis 2004; 9: 55-66.

15. Fumarola C, Zerbini A, Guidotti GG. Glutamine deprivation-mediated cell shrinkage induces ligand-independent CD95 receptor signaling and apoptosis. Cell Death Differ 2001; 8: 1004-1013.

16. Bortner $\mathrm{CD}$, Cidlowski JA. Uncoupling cell shrinkage from apoptosis reveals that $\mathrm{Na}+$ influx is required for volume loss during programmed cell death. J Biol Chem 2003; 278: 39176-39184.

17. De Almeida CJ, Linden R. Phagocytosis of apoptotic cells: a matter of balance. Cell Mol Life Sci 2005; 62: 1532-1546.

18. Chiarugi A. 'Simple but not simpler': toward a unified picture of energy requirements in cell death. FASEB J 2005; 19: 1783-1788.

19. Lane JD, Allan VJ, Woodman PG. Active relocation of chromatin and endoplasmic reticulum into blebs in late apoptotic cells. J Cell Sci 2005; 118: 4059-4071.

20. Young J, Mitran S. A numerical model of cellular blebbing: a volume-conserving, fluidstructure interaction model of the entire cell. J Biomech 2010; 43: 210-220.

21. Keller $H$, Rentsch $P$, Hagmann J. Differences in cortical actin structure and dynamics document that different types of blebs are formed by distinct mechanisms. Exp Cell Res 2002; 277: 161-172

22. Hagmann J, Burger MM, Dagan D. Regulation of plasma membrane blebbing by the cytoskeleton. J Cell Biochem 1999; 73: 488-499.

23. Tinevez JY, Schulze U, Salbreux G, Roensch J, Joanny JF, Paluch E. Role of cortical tension in bleb growth. Proc Natl Acad Sci USA 2009; 106: 18581-18586.

24. Mills JC, Stone NL, Erhardt J, Pittman RN. Apoptotic membrane blebbing is regulated by myosin light chain phosphorylation. J Cell Biol 1998; 140: 627-636.

25. López-Hernández FJ, Ortiz MA, Bayon Y, Piedrafita FJ. Retinoid-related molecules require caspase 9 for the effective release of Smac and the rapid induction of apoptosis. Cell Death Differ 2004; 11: 154-164.

26. Ortiz MA, Lopez-Hernandez FJ, Bayon Y, Pfahl M, Piedrafita FJ. Retinoid-related molecules induce cytochrome $c$ release and apoptosis through activation of c-Jun $\mathrm{NH}(2)$ terminal kinase/p38 mitogen-activated protein kinases. Cancer Res 2001; 61: 8504-8512.

27. López-Hernández FJ, Ortiz MA, Piedrafita FJ. The extrinsic and intrinsic apoptotic pathways are differentially affected by temperature upstream of mitochondrial damage. Apoptosis 2006; 11: 1339-1347.

28. Burg ED, Remillard CV, Yuan JX. K+ channels in apoptosis. J Membr Biol 2006; 209 3-20.

29. Lang F, Föller M, Lang K, Lang $P$, Ritter M, Vereninov $A$ et al. Cell volume regulatory ion channels in cell proliferation and cell death. Methods Enzymol 2007; 428: 209-225.

30. Green DR, Amarante-Mendes GP. The point of no return: mitochondria, caspases, and the commitment to cell death. Results Probl Cell Differ 1998; 24: 45-61.

31. Tait SW, Parsons MJ, Llambi F, Bouchier-Hayes L, Connell S, Muñoz-Pinedo $C$ et al. Resistance to caspase-independent cell death requires persistence of intact mitochondria. Dev Cell 2010; 18: 802-813.

32. Hughes Jr FM, Cidlowski JA. Potassium is a critical regulator of apoptotic enzymes in vitro and in vivo. Adv Enzyme Regul 1999; 39: 157-171.

33. Cain K, Langlais C, Sun XM, Brown DG, Cohen GM. Physiological concentrations of $K_{+}$ inhibit cytochrome c-dependent formation of the apoptosome. J Biol Chem 2001; 276: 41985-41990. 
34. Okada Y, Maeno E, Shimizu T, Manabe K, Mori S, Nabekura T. Dual roles of plasmalemmal chloride channels in induction of cell death. Pflugers Arch 2004; 448: 287-295.

35. Leist M, Single B, Castoldi AF, Kuhnle S, Nicotera P. Intracellular adenosine triphosphate (ATP) concentration: a switch in the decision between apoptosis and necrosis. J Exp Med 1997; 185: 1481-1486.

36. Nicotera P, Melino G. Regulation of the apoptosis-necrosis switch. Oncogene 2004; 23 2757-2765.
37. Nicotera P, Leist M. Energy supply and the shape of death in neurons and lymphoid cells. Cell Death Diff 1997; 4: 435-442.

38. Tsujimoto Y. Apoptosis and necrosis - intracellular ATP levels as a determinant for cell death modes. Cell Death Diff 1997; 4: 429-434.

39. Majno G, Joris I. Apoptosis, oncosis, and necrosis. An overview of cell death. Am J Patho 1995; 146: 3-15.

40. Fink SL, Cookson BT. Apoptosis, pyroptosis, and necrosis: mechanistic description of dead and dying eukaryotic cells. Infect Immun 2005; 73: 1907-1916. 